\title{
UNEQUAL ERROR PROTECTION FOR VIDEO STREAMING OVER WIRELESS LANS USING CONTENT-AWARE PACKET RETRY LIMIT
}

\author{
Chih-Ming Chen ${ }^{1,3}$, Chia-Wen Lin' ${ }^{2}$, and Yung-Chang Chen ${ }^{1}$ \\ ${ }^{1}$ Department of Electrical Engineering, National Tsing Hua University, \\ Hsinchu, Taiwan 30013, R.O.C., ycchen@ee.nthu.edu.tw \\ ${ }^{2}$ Department of Computer Science \& Information Engineering, National Chung Cheng University, \\ Chiayi, Taiwan 621, R.O.C., cwlin@cs.ccu.edu.tw \\ ${ }^{3}$ Chunghwa Telecom Co., Ltd.
}

\begin{abstract}
In this paper, we propose a content-aware retry limit adaptation scheme for video streaming over IEEE 802.11 wireless LANs (WLANs). Video packets of different importance are unequally protected with different retry limits at the MAC layer. The loss impact of each packet is estimated to guide the selection of its retry limit. More retry numbers are allocated to packets of higher loss impact to achieve unequal error protection. Experimental results show that the proposed adaptation scheme can effectively mitigate the error propagation due to packet loss and assure the on-time arrival of packets for presentation, thereby improving video quality significantly.
\end{abstract}

\section{INTRODUCTION}

With low cost, easy deployment, and flexible connectivity, WLAN is becoming widespread and leading to fast-growing deployments in consumer homes. However, the challenges as to cope with the time-varying error rate and fluctuating bandwidth of a wireless network bring out the need of error resilient video transport. Forward Error Correction (FEC) and Automatic Retransmission reQuest (ARQ) are the two most commonly used channel coding schemes for error protection. FEC is not effective to cope with time-varying burst errors. ARQ is particularly useful to combat against packet erasure error and has been adopted in several existing packet protection methods [1][5] for video streaming over wireless networks. In [1] the authors proposed a class of packet scheduling algorithms for wireless video streaming by applying different deadline thresholds to video packets of different importance. The importance of a packet is determined by its relative position within its group of pictures (GOP) and motion-texture context. In [2], five schemes based on uniform, frame level reference, slice-level reference, motion, and motion combined with slice-level reference, are respectively proposed for loss differentiation. A packet-level multiple-description FEC (MD-FEC) was proposed in [3] to resolve the heterogeneity of client channel conditions for video multicasting over WLANs.. The conditional retransmission scheme proposed in [4] uses the concealment error and the channel condition to determine whether a packet is worthwhile to retransmit. It provides a rate-distortion analysis of the tradeoff between the saved-bits due to the reduced retransmission and the increased distortion resulting from the concealment error of not-retransmitted packets. A timestamp and frame based content-aware retry mechanism was proposed in [5] to improve video streaming over 802.11 WLANs by dynamically determining whether to send or to discard a packet based on its retransmission deadline.

In IEEE 802.11 WLAN networks, when a station wants to send data, it needs to take a backoff process to prepare for the transmission. After the data are sent, the sender will wait for an ACK from the receiver to confirm the data arrive successfully at the receiver. However, if the sender does not receive the ACK within a specified timeout interval or detects another transmission in the channel, the sender will retransmit the frame again according to the backoff rule. For any transmission, the backoff interval is uniformly chosen in $[0, C W-1]$, where $C W$ is the contention window that will be doubled at each retransmission. A packet will be dropped after its retry limit has been reached. The standard allows a default of a maximum of seven retries before the data is dropped [6].

In this work, instead of adopting a fixed retry limit, we propose a Content-Aware Retry Limit Adaptation (CA-RLA) scheme which dynamically adapts the retry limit for each packet based on its loss impact. The encoder off-line estimates the amount of error propagation caused by each packet should it be lost during transmission. This side information is stored as metadata in the streaming server for guiding the decision on the retry limit of each packet. During the on-line streaming, the CARLA scheme increases the retry limit of the packets of higher loss impacts as well as reduces the retry limit of packets of lower loss impacts so as to minimize the overall error propagation in a GOP under the delay constraint of video presentation. Compared to CAR in [5], CA-RLA minimizes the overall error propagation within a GOP by adapting retry limits of packets based on packet-level loss-impact estimates rather than the frame-level statistics used in CAR. Besides, we propose an accurate backoff time estimation scheme of each retry to provide more timing information about video presentation deadline for guiding the retry limit adaptation.

The rest of this paper is organized as follows. Section 2 presents a scheme of estimating error propagation of each packet so that different retry limit can be assigned to the packets accordingly. In Section 3, the estimation of backoff time for each retry in wireless networks is presented. The proposed CA-RLA based on the estimated backoff time is presented in Section 4. Section 5 reports the experimental results of the proposed 
algorithms and the comparison with other methods. Finally, conclusions are drawn in Section 6.

\section{ESTIMATION OF PACKET LOSS-IMPACT}

To estimate the impact of each lost packet, we first define the pixel-level loss-impact (LI) metric as the product of two parameters: PRC (Pixel Reference Count) and PCE (Pixel Concealment Error), to characterize the amount of pixel-wise error propagation as follows:

$$
L I(x, y, u)=\operatorname{PCE}(x, y, u) \times P R C(x, y, u)
$$

where $P R C(x, y, u)$ represents the frequency of pixel $(x, y)$ of frame $u$ being referenced by pixels in the following frames within a GOP in the motion-compensated prediction (MCP) process as illustrated in Fig.1. It can be calculated recursively by summing up the individual reference counts of pixels in frame $u+1$ which reference to pixel $(x, y)$ of frame $u$ in the reverse tracking order from the last frame to the first frame of a GOP as in (2), where $N_{\mathrm{GOP}}$ is the GOP size. In (3), PCE (x,y,u) denotes the norm of concealment error of pixel $(x, y)$ of frame, where $f(x, y, u)$ is the pixel value of pixel $(x, y)$ in frame $u$, assuming the zero-motion error concealment scheme is adopted.

$$
\begin{gathered}
P R C(x, y, u)=\left\{\begin{array}{cc}
\sum_{\left(x^{\prime}, y^{\prime}, u+1\right) \rightarrow(x, y, u)} P R C\left(x^{\prime}, y^{\prime}, u+1\right) & 1 \leq u<N_{G O P} \\
1 & u=N_{G O P}
\end{array}\right. \\
P C E(x, y, u)=|f(x, y, n)-f(x, y, u-1)|^{2}
\end{gathered}
$$

Fig. 1. Illustration of pixel reference count (PRC). Assume frame $N$ is the last frame of a GOP, the number in the braces indicate the PRC of a pixel.

We then use the motion information to calculate the current frame's macroblock-level error propagation by

$$
E P_{\mathrm{MB}}(u, v)=\sum_{(x, y) \in M B_{v}} L I\left(x+M V_{x}, y+M V_{y}, u-1\right)
$$

where $v$ denotes the macroblock index in a frame; $(x, y)$ denotes the pixel coordinate; $u$ represents the time index; $\left(M V_{\mathrm{x}}, M V_{\mathrm{y}}\right)$ represents the associated motion vector of pixel $(x, y)$. Finally, all $E P_{\mathrm{MB}}$ 's in one packet are summed up to estimate the packetlevel error-propagation as follows:

$$
E P_{\mathrm{pkt}}(u, q)=\sum_{v=1}^{N_{\mathrm{MB}}} E P_{\mathrm{MB}}(u, v)
$$

where $q$ denotes the packet index of a frame, and $N_{\mathrm{MB}}$ denotes the number of macroblocks in the packet.

\section{ESTIMATION OF BACKOFF TIME}

In this paper, we propose to estimate the backoff time for each retry in an IEEE 802.11 WLAN based on a mathematical analysis by adopting the Markov chain model presented in [7]. Table 1 lists the system parameters used in the analysis. These parameters are either known a priori or can be derived from other known parameters.

Table 1. System parameters defined in [7]

\begin{tabular}{|c|l|}
\hline$n$ & Number of stations contending on the channel \\
\hline$T_{\mathrm{s}}$ & time spent in a successful transmission \\
\hline$T_{\mathrm{c}}$ & time spent in a collision \\
\hline$t$ SlotTime & time of one slot defined in [6] \\
\hline$W$ & $\begin{array}{l}W=C W_{\min } \text { is the minimum contention window } \\
\text { as defined in [6] }\end{array}$ \\
\hline$m$ & $\begin{array}{l}\text { Contention window } C W \text { of the } r \text {-th retry is } C W \\
=W_{\mathrm{r}}=2^{r} C W_{\min } . C W_{\max }=2^{m} C W_{\min } . \text { When the } \\
\text { retry reaches } m, W_{r} \text { will stay constant at } C W_{\max } \\
\text { as defined in [6] }\end{array}$ \\
\hline
\end{tabular}

We assume a saturated case as discussed in [7], in which the probability for a packet to be transmitted in a slot is given by

$$
\tau=\frac{2(1-2 p)(1-p)}{(1-2 p)(W+1)+p W\left(1-(2 p)^{m}\right)}
$$

where $p$ stands for the probability of detecting the channel busy. It is also the collision probability of transmission as given in (7).

$$
p=1-(1-\tau)^{n-1}
$$

Let $P_{t r}$ denote the probability that there includes at least one transmission in a slot, and $P_{\mathrm{s}}$ the probability that a transmission is successful, as shown below:

$$
\begin{gathered}
P_{t r}=1-(1-\tau)^{n} \\
P_{s}=n \tau(1-\tau)^{n-1}
\end{gathered}
$$

According to the access mechanism of IEEE 802.11, the backoff timer of the station will count down as the medium is sensed idle, but stop counting when any transmission is detected. Therefore, at first, we define $P_{w}(k)$ as the probability of the station to finish $w$ backoff slots with $k$ slot times:

$$
P_{w}(k)=\left(\begin{array}{c}
k-1 \\
w-1
\end{array}\right) P_{t r}^{k-w}\left(1-P_{t r}\right)^{w}
$$

where $k \geq w . P_{w}(k)$ is a negative binomial variable [8] with parameter $w$ and $P_{t r}$, and has the following property:

$$
E_{k}\left[P_{w}(k)\right]=\frac{w}{\left(1-P_{t r}\right)} \text { with } \sum_{k=w}^{\infty} P_{w}(k)=1
$$

where $E_{k}\left[P_{w}(k)\right]$ represents the mean of $P_{w}(k)$ subject to $k$.

The time duration $\delta_{w}(k)$ of finishing $w$ backoff slots with $k$ slot times can be obtained using the model presented in [7]: $\delta_{w}(k)=w \cdot t$ SlotTime $+(k-w) \cdot\left[P\left(P_{s} \mid P_{t r}\right) \cdot T_{s}+P\left(P_{t r}-P_{s} \mid P_{t r}\right) \cdot T_{c}\right]$ where $t$ SlotTime, $T_{\mathrm{s}}$ and $T_{\mathrm{c}}$ are known a priori. According to (11), we can calculate the expected value $E_{k}\left[\delta_{w}(k)\right]$ as:

$$
\begin{aligned}
& E_{k}\left[\delta_{w}(k)\right]=\sum_{k=w}^{\infty} \delta_{w}(k) \cdot P_{w}(k) \\
& =w \cdot t \text { SlotTime }+\left(E_{k}\left[P_{w}(k)\right]-w\right) \cdot\left[P\left(P_{s} \mid P_{t r}\right) \cdot T_{s}+P\left(P_{t r}-P_{s} \mid P_{t r}\right) \cdot T_{c}\right] \\
& =w \cdot t \text { SlotTime }+\left(\frac{w}{\left(1-P_{t r}\right)}-w\right) \cdot\left[P\left(P_{s} \mid P_{t r}\right) \cdot T_{s}+P\left(P_{t r}-P_{s} \mid P_{t r}\right) \cdot T_{c}\right] \\
& =\Delta(w)
\end{aligned}
$$

As defined in [6], the backoff interval $w$ of any transmission is uniformly chosen in $[0, C W-1]$, where $C W=2^{r} C W_{\min }$ for the $r$ th retry. As a result, we can derive the backoff time for the $r$-th retry as follows: 


$$
t_{b f}(r)=E_{w}[\Delta(w)]=2^{r} \cdot K
$$

where

$$
K=\frac{C W_{\min }}{2} \cdot\left\{\text { tSlotTime }+\frac{P_{t r}}{\left(1-P_{t r}\right)} \cdot\left[P\left(P_{s} \mid P_{t r}\right) \cdot T_{s}+P\left(P_{t r}-P_{s} \mid P_{t r}\right) \cdot T_{c}\right]\right\}
$$

\section{CONTENT-AWARE RETRY LIMIT ADAPTATION}

Consider a video sequence with $M$ frames, inter-coded frame

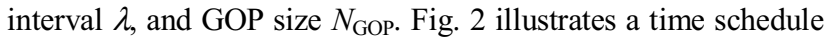
for packet transmission. We formulate the deadline of presentation for video packet $P K T_{i, j}^{q}$ as follows:

$$
D_{t}\left(P K T_{i, j}^{q}\right)=\beta+\left((i-1) \cdot N_{G O P}+(j-1)\right) \cdot \lambda
$$

where we assume an initial delay $\beta$ at the receiver, and $P K T_{i, j}^{q}$ denotes the $q$-th packet of the $j$-th frame within the $i$-th GOP. The lager the value of $\beta$ is selected, the longer retry deadline the sender can deploy, but the receiver requires a larger-size buffer and a longer delay for video presentation. We uniformly assign the initial delay $\beta$ to each GOP. According to Fig. 2, we can formulate the time period $T_{\mathrm{GOP}}$ during which all the packets of one GOP are all received at the receiver.

$$
T_{\mathrm{GOP}}=\frac{\beta+\lambda \cdot M}{M / N_{\mathrm{GOP}}}
$$

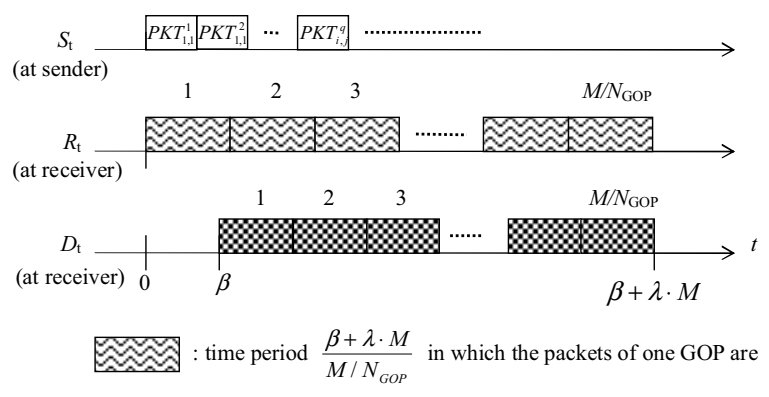

needed to be received at receiver.

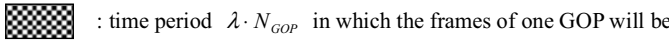
presented at receiver.

Fig. 2. Time schedule for packet transmission.

From (12), while a packet is transmitted with a retry limit $L$ and packet loss rate $P_{e}$, we can calculate the mean value of backoff time as

$$
\begin{aligned}
T\left(L, P_{e}\right)= & \left(t_{b f}(0)+T_{s}\right) \cdot\left(1-P_{e}\right) \\
& +\left(t_{b f}(0)+T_{c}+t_{b f}(1)+T_{s}\right) \cdot P_{e}\left(1-P_{e}\right) \\
& +\left(t_{b f}(0)+T_{c}+t_{b f}(1)+T_{c}+t_{b f}(2)+T_{s}\right) \cdot P_{e}^{2}\left(1-P_{e}\right) \\
& +\ldots \\
& +\left(t_{b f}(0)+T_{c}+t_{b f}(1)+T_{c}+\ldots+t_{b f}(L-1)+T_{s}\right) \cdot P_{e}^{L-1}\left(1-P_{e}\right) \\
& +\left(t_{b f}(0)+T_{c}+t_{b f}(1)+T_{c}+\ldots+t_{b f}(L-1)+T_{c}+t_{b f}(L)\right) \cdot P_{e}^{L} \\
= & \left(1-P_{e}^{L}\right) \cdot\left(T_{s}+\frac{P_{e}}{1-P_{e}} \cdot T_{c}\right)+\sum_{r=0}^{L} P_{e}^{r} \cdot t_{b f}(r)
\end{aligned}
$$

We assume the wireless link is a memoryless packet erasure channel and the packets are dropped independently. Since the $k$ - th packet is dropped after $L^{(k)}$ unsuccessful retries, we can obtain the packet erasure rate as

$$
P L R^{(k)}\left(L^{(k)}, P_{e}\right)=P_{e}^{L^{(k)}+1}
$$

Let $P L R^{(k)}$ denote the packet loss probability of the $k$-th packet in a GOP with retry limit $L^{k}$, and $E P_{\mathrm{pkt}}^{(k)}$ its packet-level error-propagation as in (5). With the delay constraint, our goal is to find a set of retry limits $\left\{L^{(1)}, L^{(2)}, \ldots, L^{(k)}, \ldots, L^{\left(N_{\mathrm{GOP}}^{\mathrm{pkt}}\right)}\right\}$ for the packets in a GOP to minimize the total error propagation of the GOP as follows:

$$
\begin{gathered}
\min _{L^{(1)}, L^{(2)}, \ldots, L^{(N) \mathrm{POP}}}\left\{\sum_{k=1}^{N N_{\mathrm{GOP}}^{\mathrm{pkt}}} P L R^{(k)} \cdot E P_{\mathrm{pkt}}^{(k)}=\sum_{k=1}^{N_{\mathrm{GOP}}^{\mathrm{pkt}}} P_{e}^{L^{(k)}+1} \cdot E P_{\mathrm{pkt}}^{(k)}\right\} \\
\text { subject to } \sum_{k=1}^{N_{\mathrm{GOP}}^{\mathrm{pkt}}} T\left(L^{(k)}, P_{e}\right) \leq T_{\mathrm{GOP}}
\end{gathered}
$$

where $N_{\mathrm{GOP}}^{\mathrm{pkt}}$ denotes the number of packets in a GOP. Table 2 shows the typical system parameters used for FHSS, where the propagation delay of data transmission is relatively negligible compared to the other system parameters.

Table 2. IEEE 802.11 system parameters for FHSS

\begin{tabular}{|c|l|}
\hline Channel bit rate & $1 \mathrm{Mbps}$ \\
\hline Propagation delay & $1 \mathrm{us}$ \\
\hline tSlot time & $50 u \mathrm{~s}$ \\
\hline SIFS & $28 \mathrm{us}$ \\
\hline DIFS & $128 \mathrm{us}$ \\
\hline
\end{tabular}

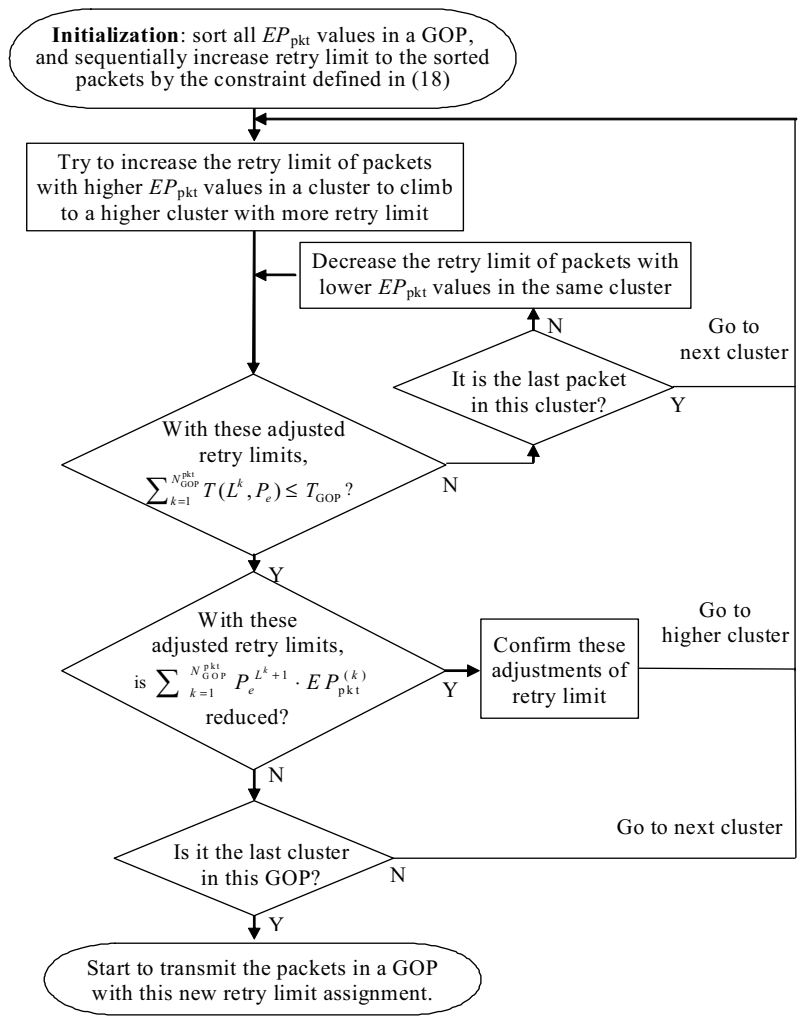

Fig. 3. Flowchart of CA-RLA. 
Based on the formulation shown in (17), the flowchart of our proposed CA-RLA algorithm is depicted in Fig.3. The proposed CA-RLA tries to increase the retry limit of the packets with higher $E P$, and to reduce the retry limit of packets with lower $E P$ under the delay constraint $T_{\mathrm{GOP}}$ and the requirement to minimize total $E P$ in a GOP.

\section{SIMULATION RESULTS}

We use the OPNET network simulator to simulate the network configuration which includes an independent basic service set (IBSS) and six stations. In the test scenario, station 1 sends an MPEG-4 video stream to station 2, while the other stations generate background traffic packets with a geometric distribution with parameter $\lambda=1$ to contend for the channel. The packet size is 160 bytes. QCIF (176x144) test sequences of 300 frames are pre-encoded at $30 \mathrm{fps}$ and $384 \mathrm{Kbps}$. The group of pictures $(\mathrm{GOP})$ size is $\left(N_{\mathrm{GOP}}, M\right)=(30,2)$, where $M$ is the distance between two anchor frames. Each row of coded macroblocks as a slice are encapsulated into one packet. According to the retry limit, a packet will be transmitted over and over until a transmission gets through or it reaches its retry limit. A packet will also be dropped in the case that the packet arrival time is later than its presentation deadline. The initial delay $\beta$ is set to $1 \mathrm{~s}$.
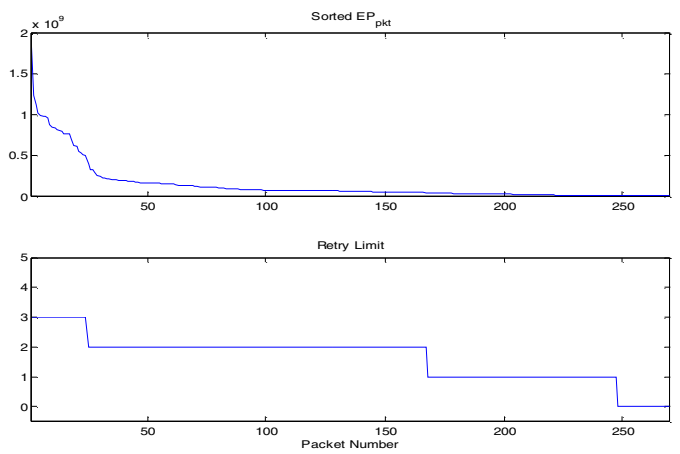

Fig. 4. Example of sorted $E P_{\text {pkt }}$ of packets in a GOP (upper), and the retry limit assigned to the packets by CA-RLA (lower).

Fig. 4 depicts an example of the sorted $E P_{\text {pkt }}$ values of all packets in a GOP and the retry limits assigned to the packets by CA-RLA. The retry limits assigned to the packets range from 0 to 3 depending on their $E P_{\text {pkt }}$ values. Fig. 5 shows the PSNR performance comparison of CA-RLA, the fixed retry limit method, and our implementation of the CAR method proposed in [5]. The results show that the method of fixed 2-retry limit causes excessive packet losses due to insufficient numbers of retries, thereby degrading the video quality severely. On the other hand, the fixed 3-retry limit leads to a relatively large number of packets being dropped due to timeout for presentation, although there are almost no packets dropped due to an insufficient number of retries. The CAR method, without taking into account the importance of each packet, tends to drop the packets closer to the end of GOP based on the retransmission deadline adaptation. Our method takes into account the importance of each retransmitted packet and the estimated backoff time of each retry for MAC-layer retry number adaptation, thereby being able to recover video quality quickly from packet losses without causing severe error propagation.

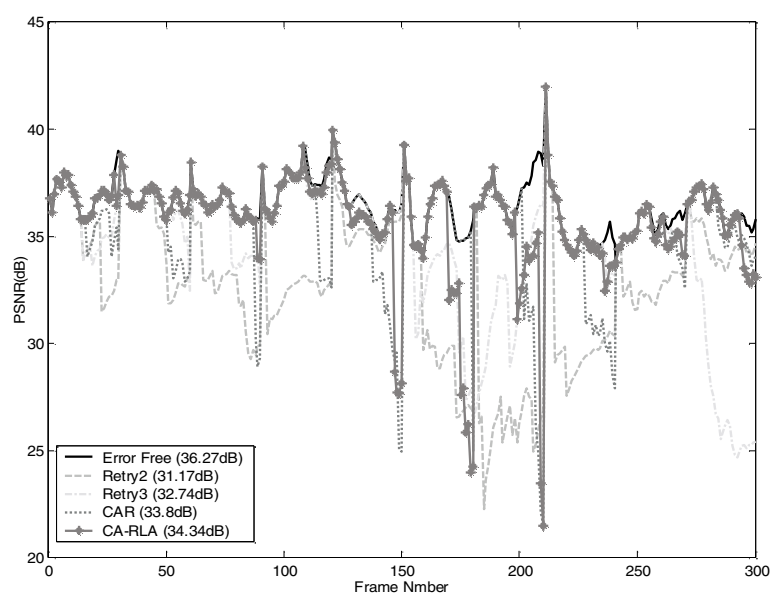

Fig. 5. Frame-by-frame PSNR performance comparison with other methods.

\section{CONCLUSION}

We have proposed a novel CA-RLA scheme to adaptively set the retry limits of packets according to its error propagation characteristics for video streaming over WLANs. The CA-RLA scheme analyzes the backoff time for each retry, so as to find a retry limit set for packets in a GOP to minimize the total errorpropagation of the GOP according to the delay constraint of packets for presentation at the receiver. Simulation results show that the proposed scheme outperforms the conventional fixed retry limit mechanism in terms of packet loss and visual quality of streaming video.

\section{REFERENCES}

[1] S. H. Kang and A. Zakhor, "Packet scheduling algorithm for wireless video streaming," in Proc. Workshop Packet Video, Apr. 2002, Pittsburgh.

[2] W. Tan and A. Zakhor, "Packet classification schemes for streaming MPEG video over delay and loss differentiated networks," in Proc. Packet Video Workshop, Apr. 2001, Kyongju, Korea.

[3] A. Majumdar, D. Sachs, I. Kozintsev, K. Ramchandran, and M. Yeung, "Multicast and unicast real-time video streaming over wireless LANs," IEEE Trans. Circuits Syst. Video Technol, vol. 12, no. 6, pp.524-534, June 2002.

[4] S. Aramvith, C.-W. Lin, S. Roy, and M.-T. Sun, "Wireless video transport using conditional retransmission and low-delay interleaving," IEEE Trans. Circuits Syst. Video Technol., vol. 12, no. 6, pp. 558-565, June 2002.

[5] M.-H. Lu, P. Steenkiste, and T. Chen, "Video streaming over 802.11 WLAN with content-aware adaptive retry," in Proc. IEEE Int. Conf. Multimedia and Expo, July 2005, Amsterdam, the Netherlands.

[6] IEEE P802.11. Standard for Wireless LAN Medium Access Control (MAC) and Physical Layer (PHY). Nov. 1997.

[7] M. Ergen and P. Varaiya, "Throughput analysis and admission control for IEEE 802.11a," Mobile Networks and Applications (MONET), vol. 10, pp. 705-716, Oct. 2005.

[8] S. Ross, Probability, Fifth Edition, Prentice Hall, 1998. 